\title{
ESTRUCTURAS SINTÁCTICAS Y LÉXICAS DE CRÓNICAS ORALES DE AJEDREZ
}

\section{SYNTACTIC AND LEXICAL STRUCTURES OF CHESS NARRATIVES}

Sara Quintero Ramírez

Universidad de Guadalajara, México.

sara.quintero@academicos.udg.mx

\section{Resumen}

En este artículo nos proponemos identificar y caracterizar la terminología y las estructuras sintácticas más prolíficas en crónicas orales de partidas de ajedrez. Para ello, nos basamos en un corpus constituido de la crónica de cuatro eventos ajedrecísticos con su respectiva transcripción. A partir de investigaciones realizadas respecto al discurso deportivo, así como en estudios relativos al ajedrez, distinguimos los términos y las estructuras utilizadas en los diferentes momentos de la crónica ajedrecística y determinamos la función que despliegan a nivel discursivo. Con este artículo nos proponemos contribuir a los estudios sobre discurso deportivo, en especial aquellos enfocados en las crónicas de disciplinas deportivas de predominio intelectual como el ajedrez.

Palabras clave: crónica deportiva, discurso ajedrecístico, medios digitales, términos, estructuras sintácticas.

\begin{abstract}
In this article, we identify and characterize the syntactic structures and terminology more frequently used in oral narratives of chess games. In order to achieve this, we built a corpus of four different chess games with their respective transcription. Based on research about sports discourse and chess as well, we identify the structures used in the different moments of the narrative and we determine their function at the level of the discourse. With this article, we aim to contribute to the studies of sports discourse, particularly to those studies focused on intellectual sports narratives, such as chess.
\end{abstract}

Key words: sports narrative, chess discourse, digital media, terms, syntactic structures.

Recibido: 08-10-2019

Aceptado: 09-11-20

\section{INTRODUCCIÓN}

El deporte se ha establecido desde hace ya bastante tiempo como una manifestación cultural trascendental que se encarga de integrar personas de diferentes países, posiciones sociales y creencias (Arias 35). Esta manifestación cultural es tan importante al grado de que no solo existen una terminología y estructuras propias de los deportes en general y de cada disciplina deportiva (Kowalikowa 64), sino que el discurso deportivo es considerado un lenguaje sectorial (Medina 197-198; Saiz 197), ya que los lenguajes 
sectoriales suelen tener un elemento sociológico que se evidencia mediante los campos de las profesiones, así como de las actividades específicas.

we can talk about sports terminology, which is now not only an integral part of the culture, but also is the sphere of professional activity, about the existence of sports terminological system, the core of which consists of common to all (or many) sports terms, designating competition, participants, the elements of the organization, constituent to the various terminological systems of the lowest level practically without changes of the notion contents (Karayev 311).

Si bien existen términos comunes en los deportes en general, hay algunas disciplinas que resultan más difíciles de entender que otras por la terminología propia que las encripta y las torna hasta cierto punto incomprensibles para la gente que no está familiarizada con ellas (Saiz 197; Gómez 151; Alasalmi 7).

Ahora bien, aunque el discurso deportivo ha sido objeto de estudio de un sinnúmero de investigaciones lingüísticas, no todos los deportes han recibido la misma atención. En efecto, existen múltiples trabajos enfocados en el análisis del discurso de deportes etiquetados como 'populares' tales como el futbol (Medina; Gómez; Saiz), el béisbol (Castro), el boxeo (Armañanzas y Sánchez), el rugby (Mathon y Boulakia; Augendre et al.), entre otros. Empero, pocos son los estudios que examinan el discurso de disciplinas deportivas menos físicas y más intelectuales, como es el caso específico del discurso ajedrecístico (Maksymenko 91). Si bien existe una inmensa bibliografía relacionada con el ajedrez, el número de obras se torna exiguo al tratarse de investigaciones de corte lingüístico.

Con base en los hechos antes descritos, planteamos el objetivo del presente artículo que consiste en identificar y caracterizar las estructuras léxicas y sintácticas empleadas por los cronistas de eventos ajedrecísticos. Con este manuscrito pretendemos contribuir a los estudios relativos al discurso deportivo, sobre todo para sentar las bases de investigaciones en idioma español que se enfoquen en la descripción sintáctica de crónicas orales de disciplinas deportivas no prototípicamente físicas, como el ajedrez.

Para presentar este estudio, en primer lugar, se exponen fundamentos teóricos que dan cuenta de lo que es el ajedrez, así como de sus características principales a nivel discursivo. Enseguida, en el apartado de la metodología, se describe el corpus de la investigación y la manera en la que se ha examinado. Posteriormente, nos ocupamos de 
analizar dicho corpus a fin de alcanzar el objetivo propuesto. Por último, se presentan las conclusiones derivadas de la presente investigación.

\section{FUNDAMENTOS TEÓRICOS}

\subsection{El ajedrez como deporte}

El ajedrez es considerado un juego de mesa popular que desafía al intelecto y cuyos orígenes se remontan siglos atrás (Karayev 311). Ermiş y Erilli (221) definen el ajedrez como un juego intelectual complejo que requiere de habilidades sofisticadas para resolver problemas. Kovacic (30) señala que el ajedrez es un poderoso instrumento para el desarrollo de la inteligencia y de competencias transversales en la edad escolar. De acuerdo con Bandera Castro (49-50), el ajedrez es un deporte de predominio mental cuyos jugadores deben tener conocimiento sobre la técnica ajedrecista, dominar las estrategias, así como las acciones tácticas defensivas y ofensivas durante una partida. Por su parte, Hesse concibe el ajedrez como:

un arte marcial intelectual y a la vez un campo de pruebas para la estética, la pasión y el heroísmo intelectual, un completo reino de ideas, emociones y fantasías, y también perspicacia, estimulación mental de los hemisferios izquierdo y derecho del cerebro, creatividad conjunta y una maravillosa armonía entre elementos lógicos y paradójicos (Hesse 11).

Como podemos apreciar en los párrafos anteriores, el ajedrez es definido de muy diversas maneras. Para Karayev y Ermiş y Erilli, se trata de un juego; para Hesse, es un arte marcial cerebral; mientras que, de acuerdo con Kovacic, se concibe como un instrumento para el desarrollo de competencias. Por último, para Bandera Castro, es una disciplina deportiva.

Coincidimos con Bandera Castro, ya que el Comité Olímpico Internacional (COI) designó a la Federación Internacional de Ajedrez (FIDE) como una organización deportiva mundial desde 1999, por varias razones entre las que destacan: a) su accesibilidad, pues la FIDE rechaza cualquier tipo de discriminación; b) su exigencia de rendimiento físico hacia los ajedrecistas, porque produce desgaste del sistema nervioso y cardiovascular; c) su organización a través de normas y reglas específicas; d) su concepción como competencia, pues hay torneos locales, nacionales, internacionales y 
mundiales, como en muchas disciplinas deportivas (Santoro 4-6; Fernández Amigo 99; Blanco Hernández 6-10).

Además de las características que despliega como disciplina deportiva, "el ajedrez tiene su propia estética, al generar obras notables que constituyen verdaderas muestras de expresión artística por su belleza, armonía y síntesis de alto intelecto humano; obras indestructibles que el paso del tiempo las hace cada vez más vigorosas" (Blanco Hernández 4).

\subsection{Discurso ajedrecístico}

De acuerdo con Maksymenko (91), si bien el discurso del ajedrez forma parte del discurso deportivo, esta disciplina posee una terminología, una sintaxis y una semántica especiales derivadas esencialmente de su procedimiento de juego, así como de sus reglamentos. Según el autor, para una adecuada comprensión del discurso ajedrecístico, es necesario el conocimiento del ajedrez, especialmente de su léxico.

Karayev (312) señala que la terminología que da cuenta de las partidas de ajedrez se ha formado de diversas maneras. Primeramente, existen los préstamos de otras lenguas como en el caso de blitz o en prise. Igualmente, advertimos fenómenos de derivación a través de sufijos, como centralización, combinación y coronación en este sistema terminológico. Por último, se encuentran los sintagmas ya lexicalizados como captura al paso o peón envenenado.

Además del vocabulario propio del ajedrez, al momento de dar cuenta de los movimientos realizados por los dos jugadores de una partida se requiere de una notación descriptiva $^{1}$ presentada como una combinación de letras y números que van desde la $a$ hasta la $h$ para las filas y del 1 al 8 para las columnas a fin de describir los movimientos de las piezas en cada uno de los sesenta y cuatro escaques del tablero de ajedrez (Maksymenko 92).

Lo anterior nos lleva a considerar el discurso ajedrecístico como un tipo especial de discurso deportivo cuyos participantes pertenecen a una determinada comunidad discursiva (Maksymenko 93). No obstante, el discurso ajedrecístico retoma ciertos aspectos del discurso deportivo en general (Karayev 311). Dichos aspectos no son solamente a nivel del léxico o de las estructuras, sino también a nivel de los géneros

\footnotetext{
${ }^{1}$ Además de la notación descriptiva, existe la notación algebraica.
} 
discursivos que se dedican a dar cuenta del ajedrez como la crónica, el comentario y la entrevista que son comunes para diversos deportes (Augendre et al. 209).

\subsection{Crónica deportiva oral}

Como el objeto de estudio de la presente investigación es la crónica oral de eventos ajedrecísticos, nos parece pertinente definir la crónica deportiva en general. En numerosas disciplinas deportivas, la crónica oral, ya sea a través de radio, televisión o medios digitales, consiste en relatar las acciones más relevantes que van ocurriendo en el evento deportivo de forma casi inmediata (Armañanzas y Sánchez 6).

La crónica deportiva es considerada tanto un género referencial como uno expresivo (Vega Jiménez 93). En efecto, Mathon y Boulakia (287) advierten que la crónica de deportes físicos, como el rugby y el futbol, está constituida de dos fases, una de carácter descriptivo-narrativo y otra de comentario. En la primera, el cronista relata y describe los hechos más destacados. En la segunda, el cronista abunda sobre un momento particular del evento, ofrece datos históricos, estadísticos o culturales y opina respecto a lo relatado. En esta investigación, nos proponemos determinar las estructuras sintácticas y léxicas que se producen en estas dos fases de la crónica ajedrecística.

Asimismo, en la crónica de cualquier evento deportivo, es imperante que el cronista sea experto de la disciplina narrada (Marín 257). Por un lado, debe actualizarse sobre los cambios en los reglamentos de los torneos. Por otra parte, debe investigar quiénes son los posibles portentos de la disciplina en cuestión. Su principal función en la crónica consiste en proveer un mensaje claro y preciso para que el receptor pueda formar su propia opinión (Godoy 16).

De acuerdo con Herrero (120), el léxico utilizado en la crónica deportiva oral debe ser claro, rico y entendible. Empero, el deporte en general y las disciplinas deportivas en particular explotan cierta terminología especializada que es utilizada en los diferentes medios de comunicación por ser fenómenos de masas (Nomdedeu 92). Herrero (121122) advierte que este léxico especializado puede estar constituido de extranjerismos, onomatopeyas, sobrenombres y términos metafóricos, sobre todo de carácter bélico.

En cuanto a las estructuras, estas suelen ser ricas y diversas para amenizar el discurso. Dichas estructuras suelen ser atractivas para mantener el interés del espectador (Guerrero, 367). Cuando se da cuenta de los hechos del partido, el cronista se sirve de 
construcciones verbales en presente de indicativo (Herrero 123) para transmitir de manera veraz el ritmo de un partido, así como la fuerza y la habilidad de los atletas (Hernández 158). Para referir a los protagonistas deportivos, los cronistas tienden a recurrir a una diversidad de estructuras metonímicas que despliegan algún rasgo característico del atleta en cuestión (Quintero Metonimia 282).

\subsection{Antecedentes de nuestro estudio}

Como hemos comentado anteriormente, la literatura enfocada en el discurso ajedrecístico no es tan cuantiosa como sucede con los estudios relativos al discurso de otras disciplinas deportivas. A continuación, damos cuenta de algunas investigaciones centradas en el lenguaje desarrollado en este deporte.

A manera de ejemplo, Simó realiza un análisis contrastivo de las expresiones metafóricas para describir un juego de ajedrez en inglés americano y húngaro. Con base en corpus de notas periodísticas, artículos de revistas y sitios web ajedrecísticos, la autora se centra en metáforas pertenecientes al campo semántico de las partes del cuerpo y advierte que en ambas lenguas se utilizan expresiones metafóricas variadas.

Por su parte, Karayev señala la necesidad de encontrar el significado del metalenguaje de los términos empleados en el ajedrez a fin de tener una comprensión más integral del fenómeno en cuestión. La autora advierte que la terminología utilizada en el ajedrez incluye términos coloquiales, humorísticos, nominales y verbales que dan cuenta de lo que sucede en el tablero.

Asimismo, en idioma ruso, encontramos dos investigaciones que examinan el discurso del ajedrez. Por un lado, Rakhimova se centra en el análisis terminológico del ajedrez contrastando textos escritos profesionales, el libro de Karpov, y textos de ficción, la novela de Roshal. Por otro lado, Maksymenko considera el discurso ajedrecístico como una variedad del discurso deportivo que se caracteriza por tener un modo particular y un estilo funcional.

Por último, Palacios Aldana y Mengana Osorio se enfocan en la comunicación que se produce en conversaciones, charlas y explicaciones al momento de enseñar ajedrez. De acuerdo con las autoras, la interactividad que se produce entre los participantes de un curso de ajedrez juega un rol determinante para hacer del discurso ajedrecístico un lenguaje con rasgos particulares. 
Los antecedentes que hemos identificado nos permiten aseverar que el lenguaje empleado en distintas realidades ajedrecísticas, por ejemplo: partidas, libros de enseñanza, libros de ficción y clases presenciales, despliega una sintaxis y una semántica específicas que se originan de sus tácticas, reglas y maniobras. Empero, la originalidad de nuestro estudio radica en el análisis de las estructuras sintácticas y léxicas que se explotan en la crónica ajedrecística oral en español.

\section{Metodología}

\subsection{Conformación del corpus}

En el presente estudio, nos basamos en un corpus de cuatro crónicas orales de partidas de ajedrez en diferentes torneos. Consideramos dos partidas de ritmo clásico y otras dos partidas blitz. Es importante advertir que, a grandes rasgos, las partidas de ritmo clásico se caracterizan porque cada jugador cuenta con más de sesenta minutos para desarrollar sus movimientos; mientras que en las partidas blitz cada jugador cuenta con menos de diez minutos (cf. FIDE).

En el caso de las partidas de ritmo clásico, consideramos, por un lado, la división de honor del Campeonato de España por equipos que se celebró en Melilla entre Ivan Cheparinov y Vassily Ivanchuk el 30 de septiembre de 2019. Por otro lado, tomamos en cuenta una partida entre Magnus Carlsen y Ding Liren correspondiente a la octava ronda del Grand Chess Tour de Croacia que tuvo lugar el 4 de julio de 2019 en Zagreb. Ambas partidas fueron comentadas por José Fernando Cuenca Jiménez, conocido como Pepe Cuenca, y David Martínez Martín, “El Divis”.

En el caso de las partidas blitz, consideramos la partida entre Maxim Matlakov y Magnus Carlsen, perteneciente al Campeonato del Mundo de Ajedrez Blitz 2019 celebrado en Moscú el 29 de diciembre. Asimismo, tomamos la partida entre Magnus Carlsen y Ding Liren correspondiente a los desempates de la Sinquefield Cup que tuvo lugar el 9 de septiembre de 2019 en San Luis, Misuri. Ambas partidas fueron narradas por Luis Fernández Siles, “Luisón”.

Las crónicas de las partidas fueron tomadas de su respectiva transmisión por medios digitales. Por un lado, las dos partidas con ritmo clásico se recogieron de Chess 24 en Español. Por otro lado, las partidas blitz se tomaron de Chess.com ES. Las características más importantes de cada partida del corpus se muestran en la tabla 1. 


\begin{tabular}{|c|c|c|c|c|}
\hline Contendientes & Evento deportivo & Lugar y fecha & Ritmo & $\begin{array}{l}\text { Medio de } \\
\text { difusión }\end{array}$ \\
\hline $\begin{array}{l}\text { Ivan Cheparinov vs } \\
\text { Vassily Ivanchuk }\end{array}$ & $\begin{array}{l}\text { División de honor del } \\
\text { Campeonato de } \\
\text { España por equipos }\end{array}$ & $\begin{array}{ll}\text { Melilla, } 30 & \text { de } \\
\text { septiembre } & \text { de } \\
2019 & \end{array}$ & Clásico & $\begin{array}{l}\text { Chess24 en } \\
\text { Español }\end{array}$ \\
\hline $\begin{array}{l}\text { Magnus Carlsen vs } \\
\text { Ding Liren }\end{array}$ & $\begin{array}{l}\text { Octava ronda del } \\
\text { Grand Chess Tour de } \\
\text { Croacia } 2019\end{array}$ & $\begin{array}{l}\text { Zagreb, } 4 \text { de } \\
\text { julio de } 2019\end{array}$ & Clásico & $\begin{array}{l}\text { Chess24 en } \\
\text { Español }\end{array}$ \\
\hline $\begin{array}{l}\text { Maxim Matlakov } \\
\text { vs Magnus Carlsen }\end{array}$ & $\begin{array}{l}\text { Campeonato } \quad \text { del } \\
\text { Mundo de Ajedrez } \\
\text { Blitz } 2019\end{array}$ & $\begin{array}{l}\text { Moscú, } 29 \text { de } \\
\text { diciembre } \\
2019\end{array}$ & Blitz & $\begin{array}{l}\text { Chess.com } \\
\text { ES }\end{array}$ \\
\hline $\begin{array}{l}\text { Magnus Carlsen vs } \\
\text { Ding Liren }\end{array}$ & $\begin{array}{l}\text { Desempates de la } \\
\text { Sinquefield Cup } 2019\end{array}$ & $\begin{array}{l}\text { San Luis, Misuri, } \\
9 \text { de septiembre } \\
\text { de } 2019\end{array}$ & Blitz & $\begin{array}{l}\text { Chess.com } \\
\text { ES }\end{array}$ \\
\hline
\end{tabular}

Tabla 1. Descripción de las crónicas ajedrecísticas del corpus

Cabe señalar que hemos elegido las crónicas de los medios digitales antes aludidos, porque dichos medios son los únicos a los que se tiene acceso a las crónicas de partidas de ajedrez a nivel mundial en gran parte de los países de habla hispana.

\subsection{Procedimiento de análisis}

Una vez que seleccionamos las cuatro partidas del corpus de nuestro estudio, transcribimos cada una, tal como fueron transmitidas por los medios digitales antes aludidos. Luego de hacer la transcripción, procedimos a identificar las etapas de la crónica con base en la propuesta de Mathon y Boulakia (287), esto es: a) fase descriptivo-narrativa y b) fase de comentario.

Posteriormente, con base en las dilucidaciones de Maksymenko (91-93) y Karayev (311-312), examinamos cada fase de las cuatro crónicas con la finalidad de reconocer tanto las estructuras como los términos producidos con mayor asiduidad por los cronistas del corpus. Enseguida, nos dimos a la tarea de determinar la función específica que desempeñan dichas estructuras léxicas y sintácticas a nivel del discurso. 
Por último, contrastamos nuestros resultados con aquellos de otros estudios enfocados en crónicas de diversas disciplinas deportivas a fin de poder establecer semejanzas y diferencias entre la crónica ajedrecística y aquella de deportes más físicos.

\section{ANÁLISIS}

Con base en Mathon y Boulakia (287), observamos que la crónica de las partidas de ajedrez de nuestro corpus está conformada básicamente de dos etapas, una en la que los responsables de la crónica se ocupan de dar cuenta de lo que está ocurriendo en el tablero de ajedrez: etapa descriptivo-narrativa, y otra en la que los cronistas comentan y ofrecen opiniones respecto a cuestiones relacionadas con el juego: etapa de comentario.

En las cuatro crónicas que conforman el corpus de nuestro estudio, advertimos que la fase de comentario es, por mucho, la más explotada por los cronistas ajedrecísticos. Consideramos que esto es comprensible dada la naturaleza de las partidas de ajedrez en las que los movimientos desarrollados en el tablero no se realizan con tanta rapidez, en las partidas de ritmo clásico y de blitz, como para que el cronista solamente se enfoque en la descripción-relato de estos.

Una vez identificadas las fases, a continuación, nos proponemos examinar las estructuras y los términos más iterativos en las crónicas del corpus a fin de cotejarlos con las elucidaciones de los estudiosos del lenguaje del ajedrez. Damos inicio con la fase descriptivo-narrativa.

\subsection{Fase descriptivo-narrativa}

A grandes rasgos, observamos que la fase enfocada en relatar las jugadas se caracteriza por la producción de estructuras sintácticas cortas. Muchas de estas estructuras tienden a ser construcciones averbales. De acuerdo con Mathon y Boulakia, en esta fase de la crónica, es común encontrar este tipo de construcciones:

On trouve ainsi dans le commentaire sportif et plus particulièrement dans la partie descriptive un grand nombre de ces constructions, non pas qu'elles soient propres au genre, mais elles sont particulièrement présentes. Ces constructions ont l'avantage de s'adapter facilement aux aléas du discours qui suit les aléas de l'action. Par ailleurs, dans cette partie descriptive, le locuteur doit fournir une part d'informations au téléspectateur, pour qu'il puisse suivre l'action, y compris dans sa compréhension des techniques de jeu (Mathon y Boulakia 287-288). 
En el marco de las construcciones averbales, advertimos la presencia de sintagmas nominales cuya función consiste en la descripción del movimiento que ejecuta el jugador en turno, como se constata en (1), así como la designación del ajedrecista que mueve las piezas y el movimiento en sí, como se observa en (2).

(1) $[\ldots]$ e4, d5, siciliana d6, f4. [Cheparinov vs Ivanchuk - Campeonato de España por equipos]

(2) Maxim Matlakov hacia h4. [Matlakov vs Carlsen - Campeonato del Mundo de Ajedrez Blitz]

Las construcciones averbales no son las únicas que se producen en la fase descriptivonarrativa, pues también registramos construcciones verbales. Estas se caracterizan por presentar la jugada mediante verbos como enrocarse o decidir/ definir/ jugar + un sintagma nominal que refiere al movimiento ejecutado, así como la designación de la persona que ha realizado el movimiento, tal como se aprecia en (3) y (4).

(3) Ya se enrocó Cheparinov. [Cheparinov vs Ivanchuk - Campeonato de España por equipos]

(4) [...] una vez que Magnus Carlsen decide definir el centro, juega d5. [Matlakov vs Carlsen - Campeonato del Mundo de Ajedrez Blitz]

En esta fase descriptivo-narrativa, las estructuras que dan cuenta de los movimientos realizados por los jugadores están constituidas esencialmente del nombre de la pieza y del escaque al que se ha desplazado la pieza en cuestión, como se advierte en (5-8). Como lo señala Maksymenko (92), la designación del escaque se hace a través de una letra para las filas y un número para las columnas. Si bien en la notación descriptiva ajedrecística lo adecuado es escribir la letra con la que se nombra la pieza que se mueve en mayúscula junto con la coordenada de destino (letras minúsculas y números), nosotros optamos por escribir el nombre de la pieza completo, porque así fue enunciado por el cronista del corpus.

(5) Alfil e2, fuera enroque. [Cheparinov vs Ivanchuk - Campeonato de España por equipos]

(6) Ding Liren reacciona rápidamente caballo a5 desalojando al alfil de b3, c5 para ganar mayor presencia central. [Carlsen vs Liren Sinquefield Cup 2019]

(7) Después de dama b5, las negras proponen un cambio de dama. [Carlsen vs Liren - Sinquefield Cup 2019] 
(8) Por fin [Carlsen] juega caballo f6. [Matlakov vs Carlsen Campeonato del Mundo de Ajedrez Blitz]

Asimismo, cuando se trata del movimiento de un peón, el cronista solamente enuncia el nombre de la casilla a la que el jugador desplaza dicho peón mediante la letra y el número correspondientes, como se observa en (9) y (10).

(9) Y bueno por fin le juegan $d 4$ a Magnus. [Carlsen vs Liren - Grand Chess Tour]

(10) Las blancas que deciden jugar con un rápido $d 4$, a pesar de que al inicio ya jugó $d 3$. [Carlsen vs Liren - Sinquefield Cup 2019]

Otro rasgo iterativo en esta fase descriptivo-narrativa es aludir a la serie de movimientos ejecutados a través de los nombres con los que se conocen diversas jugadas en el mundo ajedrecístico, como sucede en (11-14): catalana, siciliana, grand prix, gambito de dama, semieslava, Cambridge Spring, apertura española, línea del dragón, entre otras. Cabe señalar que tanto la catalana como la española y el gambito de dama hacen referencia a aperturas; mientras que la siciliana, la semieslava y la Cambridge Srping son defensas, es decir la reacción de las piezas negras ante el movimiento de las blancas. Asimismo, la línea del dragón es una variante de la defensa siciliana. Por último, un grand prix hace referencia a un ataque contra la defensa siciliana. Los nombres de las jugadas pueden mencionarse tanto en el marco de sintagmas nominales, como sucede en (11) y (12), así como en construcciones verbales, como en (13-15).

(11) Hombre, catalana de Ding, de primera Liren. [Carlsen vs Liren Grand Chess Tour]

(12) e4, d5, siciliana d6, f4, un grand prix. [Cheparinov vs Ivanchuk Campeonato de España por equipos]

(13) Bueno, se traspone un gambito de dama, las negras juegan la semieslava con c6 y parece que esto traspone a una Cambridge Spring. [Matlakov vs Carlsen - Campeonato del Mundo de Ajedrez Blitz]

(14) Eso sí con las piezas blancas plantea una apertura española. [Carlsen vs Liren - Sinquefield Cup 2019]

(15) Pero ahora la hace $\mathrm{d} 4$, con lo que han ido a parar justo una línea del dragón. [Cheparinov vs Ivanchuk - Campeonato de España por equipos]

Asimismo, tal como lo comenta Maksymenko (91), en la crónica ajedrecística, es común que los sustantivos negras y blancas se utilicen con frecuencia para designar a los ajedrecistas que juegan con las figuras del color correspondiente, como sucede en 
los fragmentos de (16) y (17). En otras palabras, se trata de un recurso metonímico prototípico de la crónica de casi todas las disciplinas deportivas (cf. Quintero Metonimia).

(16) Bueno, se traspone un gambito de dama, las negras juegan la semieslava con c6 y parece que esto traspone a una Cambridge Spring. [Matlakov vs Carlsen - Campeonato del Mundo de Ajedrez Blitz]

(17) Y las blancas que deciden jugar con un rápido d4, a pesar de que al inicio ya jugó d3. [Carlsen vs Liren - Sinquefield Cup 2019]

Cabe señalar que los sustantivos antes aludidos no son las únicas metonimias utilizadas por los cronistas ajedrecísticos. En efecto, tal como sucede en las crónicas de otras disciplinas deportivas, en esta se menciona alguna característica prototípica de los ajedrecistas o incluso algún logro particular para aludir a ellos (Quintero Metonimia 282), tal como se aprecia en (18) y (19), donde Magnus Carlsen es referido por su calidad de campeón del mundo (18), así como por su nacionalidad (19). Respecto de este último fragmento (19), el cronista no solo recurre a la metonimia de la nacionalidad, sino que además la hace acompañar de una metáfora, a través del término héroe, para aludir al ajedrecista en cuestión.

(18) Se rinde Matlakov. ¡Gran partido del campeón del mundo! [Matlakov vs Carlsen - Campeonato del Mundo de Ajedrez Blitz]

(19) Vamos a ver cómo quiere completar el desarrollo Magnus Carlsen, el héroe noruego, con alfil e6, atacando c4, ataca c4. [Matlakov vs Carlsen - Campeonato del Mundo de Ajedrez Blitz]

\subsection{Fase de comentario}

Como hemos mencionado anteriormente, la fase de comentario es la más recurrente en las crónicas de ajedrez, no solamente en las partidas de ritmo clásico, sino también en aquellas de blitz, ya que los ajedrecistas deben tomarse su tiempo para poder ejecutar las jugadas más apropiadas en sus respectivas partidas. El tiempo que toman los ajedrecistas para pensar es aprovechado por los cronistas para ofrecer comentarios y acotaciones en torno a diversas situaciones cercanas y no tan cercanas respecto a la partida.

En lo que concierne a las estructuras de la fase de comentario en el discurso ajedrecístico, observamos que estas se distinguen de aquellas de la fase descriptivo- 
narrativa esencialmente porque son mucho más extensas, además de que se producen mayormente a través de construcciones verbales. Empero, cabe señalar que las construcciones averbales no están exentas en esta fase.

Las situaciones hipotéticas son sumamente iterativas en nuestros materiales. En efecto, después de una jugada, el cronista vislumbra las posibles respuestas del ajedrecista contrincante. Con gran asiduidad, las hipótesis del corpus se producen a través de la conjunción si en la prótasis. De acuerdo con Pérez Saldanya (3303), si resulta la conjunción condicional más prototípica y admite tanto indicativo como subjuntivo dependiendo del grado de posibilidad o irrealidad con que se enuncie la hipótesis. En el ejemplo (20), además de la conjunción si, constatamos que el cronista recurre al presente del modo indicativo tanto en la prótasis como en la apódosis, porque desde su perspectiva, la situación que enuncia es muy probable que llegue a cumplirse.

(20) ¡Cuidado! Bueno, de momento, si retira el caballo, cae el peón de a2. [Matlakov vs Carlsen - Campeonato del Mundo de Ajedrez Blitz]

Asimismo, las situaciones hipotéticas se enuncian a través de construcciones averbales que siguen presentando la conjunción condicional prototípica si en la prótasis, como se advierte en el fragmento de (21).

(21) Si caballo d2, caballo e4. [Carlsen vs Liren - Grand Chess Tour] Igualmente, las situaciones hipotéticas también son presentadas por los cronistas del corpus a manera de preguntas mediante la fórmula: qué pasa si..., tal como se aprecia en el fragmento de (22).

(22) ¿Qué pasa si las negras mueven el caballo? ¿Qué pasa si las negras retiran el caballo? [Matlakov vs Carlsen - Campeonato del Mundo de Ajedrez Blitz]

Las suposiciones también son comunes en la fase de comentario de esta crónica. En los ejemplos (23) y (24), la suposición es clara mediante el verbo suponer seguido de subordinada sustantiva. Asimismo, en el fragmento de (23), después de la subordinada sustantiva encontramos otra construcción verbal que se utiliza para presentar una suposición: no creer, seguida de otra subordinada sustantiva.

(23) Supongo que las blancas comen y la verdad no creo que esto compense ceder la pareja de alfiles. [Carlsen vs Liren - Grand Chess Tour] 
(24) Así que supongo que la idea de Ivanchuk va a ser no jugar enroque, ahí lo tenemos en la cámara. [Cheparinov vs Ivanchuk Campeonato de España por equipos]

En el fragmento de (25), la suposición se realiza a través del adverbio de modalidad seguramente que es utilizado dos veces. Según Veselinović (13), este adverbio se utiliza para presentar probabilidad alta y se encuentra acompañado de construcciones verbales en modo indicativo.

(25) Seguramente, Ding Liren lo tenía previsto. Seguramente ha calculado que un sacrificio de calidad en esta posición puede funcionar. [Carlsen vs Liren - Sinquefield Cup 2019]

Asimismo, estas suposiciones se producen mediante el condicional presente (26). De acuerdo con Veselinović (10), el condicional simple se emplea para expresar una suposición, concibiendo que el hecho presentado es simplemente probable o posible.

(26) Es interesante tanto alfil por c6, a pesar de que venga dama por c3. Luego habría un alfil por d5 creando un ataque a la descubierta. [Carlsen vs Liren - Sinquefield Cup 2019]

Con menos asiduidad que las hipótesis y las suposiciones, el cronista ajedrecístico también recurre a remembranzas respecto a un jugador, como sucede en el fragmento (27), donde el cronista menciona que el ajedrecista Magnus Carlsen juega con piezas blancas en la partida que está relatando. En ese momento, hace remembranza de un torneo en el que participó el ajedrecista en cuestión, así como la manera en la que realizó la apertura contra un jugador legendario.

(27) Con las piezas blancas, Magnus Carlsen hace ya muchos años en Reikiavik contra Gary Kasparov. Entonces Magnus era un niño ahora es el campeón del mundo. En aquella partida, si no recuerdo mal, se jugó dama c2 en lugar de torre c1. [Matlakov vs Carlsen Campeonato del Mundo de Ajedrez Blitz]

Por último, como hemos mencionado anteriormente, los cronistas de las partidas de ajedrez también se permiten comentar anécdotas personales, como aquella en el fragmento (28). Este tipo de recurso tiene la función de amenizar la crónica a fin de que el auditorio se entretenga mientras se producen las jugadas en el tablero.

(28) Treinta y tres grados, pero parece un infierno, macho, en el metro de Barcelona ¿eh? En metro, Barcelona es una de las peores 
experiencias que he pasado en mi vida ¿no? Hace ya muchísimos años. Recuerdo que tenía una cita. Y bueno, pues tenía que llegar en metro ¿no? Y era en julio. Claro salí empapado de sudor y dije qué hago ahora ¿no? Era, era, daba asco, daba asco mirarme ¿no? Y ese metro de Barcelona en julio es diabólico [risas]. [Carlsen vs Liren Grand Chess Tour]

Cabe señalar que, en la fase de comentario, también encontramos algunos pasajes en los que el cronista explica las jugadas que pueden hacerse a partir del movimiento ejecutado por uno de los ajedrecistas del evento, así como aquellas jugadas que deben evitarse. Estas explicaciones tienen una función claramente didáctica, pues el cronista explica aprovechando la partida que está narrando para que el auditorio aprenda sobre las estrategias que pueden seguir en una partida con circunstancias semejantes. Ejemplo de esto se aprecia en (29) y (30), donde el comentarista incluso habla en segunda persona del singular para dirigirse al público que lo ve y lo escucha.

(29) [Alfil e2, fuera enroque]. Ahora esta línea si te hacen el enroque, se suele considerar un error por esta jugada, por dama b6. [Cheparinov vs Ivanchuk - Campeonato de España por equipos]

(30) Aquí esto es teórico y conocido desde hace tiempo, si estás en caballo b5, simplemente comes, no pasa nada, y aquí le estás haciendo un pequeño truco, dama b2, caballo por e4, y comiendo en d4. Hay jugadas para evitarlo, tipo caballo a a4, pero en realidad pues, siempre se consideró que el negro está aquí perfectamente bien. [Cheparinov vs Ivanchuk - Campeonato de España por equipos]

Por último, así como en la fase descriptivo-narrativa, advertimos con cierta frecuencia el empleo de metonimias a fin de hacer referencia a los jugadores de la partida: las negras, las blancas, el campeón mundial, etc., en la fase de comentario, además de la metonimia, advertimos un fenómeno constante de personificación. En efecto, observamos que los comentaristas les atribuyen a las piezas de ajedrez características que son propias de los seres humanos (Naranjo de Arcos 240), tal como se advierte en (31-33).

En el fragmento de (31), se recurre a las construcciones verbales no perder de vista $\mathrm{y}$ querer venir como si el alfil tuviera ojos y como si el caballo tuviera voluntad. En el ejemplo de (32), se emplea el verbo venir tanto para el alfil como para la torre, como si las piezas pudieran moverse por sí mismas. Por último, en (33), el cronista emplea el verbo comer para las piezas blancas, como si estas tuvieran boca. 
(31) De esa manera el alfil no pierde de vista ninguno de los dos flancos y la ventaja que tiene el blanco de que las negras hayan cambiado en $\mathrm{d} 4$ tan rápido es que la casilla c3 ha quedado disponible para el caballo que si más tarde quiere venir a g3 como suele ser habitual puede hacerlo vía e2. [Carlsen vs Liren - Sinquefield Cup 2019]

(32) Por lo pronto el alfil viene a $G 7$, y si la torre viene a A2, como acaba de ocurrir, el caballo lo ataca. [Matlakov vs Carlsen Campeonato del Mundo de Ajedrez Blitz]

(33) Supongo que las blancas comen y la verdad no creo que esto compense ceder la pareja de alfiles. [Carlsen vs Liren - Grand Chess Tour]

\subsection{Discusión de los resultados}

A lo largo de este análisis, se han observado semejanzas entre la crónica ajedrecística y la crónica de otras disciplinas deportivas, sobre todo en lo que compete a cuestiones generales (Karayev 311). Para empezar, la crónica de una partida de ajedrez se asemeja a la crónica de otros deportes porque está conformada de dos fases esenciales: descriptivo-narrativa y de comentario (Mathon y Boulakia 287). En la primera, el cronista se ocupa de dar cuenta de lo que sucede en la partida; mientras que, en la segunda, se ofrecen opiniones y acotaciones que giran en torno al juego.

A diferencia de la crónica de deportes físicos de ritmo más acelerado, como el futbol o el rugby, donde la fase descriptivo-narrativa ocupa gran parte del tiempo de la crónica, en una partida de ajedrez el cronista le dedica mucho menos tiempo a esta fase. Esto es comprensible, porque los movimientos ejecutados por los jugadores son mucho más lentos, reflexionados y planificados. No obstante, los cronistas de ajedrez deben construir su discurso de manera espontánea tal como sucede con la crónica de otros deportes (Augendre et al.).

Asimismo, al igual que sucede en la fase descriptivo-narrativa de la crónica de otros deportes, el cronista ajedrecístico recurre a construcciones averbales (Mathon y Boulakia 287-288); muy a menudo dichas construcciones son sintagmas nominales. En deportes más físicos, los sintagmas nominales se emplean para dar cuenta de los jugadores que tienen la posesión del balón en el terreno de juego (cf. Mathon y Boulakia 287); mientras que, en la crónica ajedrecística, estos sintagmas son utilizados para referir a los movimientos y las jugadas que se realizan en el tablero. 
Igualmente, como acontece en las crónicas de otros deportes, en la crónica ajedrecística se recurre con frecuencia a figuras retóricas como la metonimia (Quintero Metonimia 270-ss) y la personificación (Naranjo de Arcos 240). Más particularmente, en nuestros materiales observamos que la metonimia se utiliza con más recurrencia en el marco de la fase descriptivo-narrativa para hacer referencia a uno de los ajedrecistas, especialmente a través de algún rasgo característico como la nacionalidad, un logro o una hazaña; mientras que la personificación tiende a estar más presente en la fase de comentario por medio de verbos que denotan actividades características de seres humanos como no perder de vista, querer, venir, etc.

Al igual que en otros deportes, el ajedrez cuenta con terminología propia que encripta el discurso para neófitos y legos de esta disciplina. Ciertamente, los términos del ajedrez son numerosos y sin reparo alguno el cronista recurre a ellos de manera constante. Por un lado, tiende a señalar los movimientos producidos por los jugadores a través del nombre de la pieza, la letra y el número del escaque a donde el ajedrecista ha movido la pieza (Maksymenko 92). Por otro lado, también recurre al nombre técnico de las diferentes jugadas derivadas de dichos movimientos. En efecto, en el marco de cuatro fragmentos textuales breves, hemos advertido la presencia de ocho términos específicos, como catalana, siciliana, gambito de dama, semieslava, etc. Coincidimos con Sammalvuo, en cuanto a que "la comunicación experta en el ajedrez comparte algunas características con las jergas científicas y lenguajes de otros campos altamente especializados" (14).

Igualmente, otras semejanzas entre la crónica ajedrecística y de otros deportes se advierten en la fase de comentario. En primera instancia, como ocurre en la fase de comentario de otras disciplinas deportivas, como el futbol, el responsable de la crónica recuerda algún hecho importante en torno a algún jugador a fin de amenizar su crónica (Quintero Rasgos sintácticos 237). En segunda instancia, el cronista puede relatar alguna anécdota personal en esta fase, porque lo que sucede en el juego lo permite y a la vez entretiene al auditorio.

Ahora bien, lo que distingue a la crónica ajedrecística de otras crónicas es que la fase de comentario está constituida esencialmente de situaciones hipotéticas que son planteadas a manera de construcciones condicionales y preguntas. Igualmente, esta fase se caracteriza por las suposiciones que presenta el cronista respecto a las posibles jugadas que pueden producirse a raíz de un movimiento real en el tablero. 
Por último, otro rasgo distintivo que identificamos en la crónica ajedrecística es un discurso didáctico que se produce a través de explicaciones por parte del responsable de la crónica, que a la vez es un conocedor de este deporte porque con frecuencia también es un ajedrecista experimentado. Dichas explicaciones se dirigen al auditorio que sigue la transmisión mediante la segunda persona de singular que se observa tanto en la flexión verbal como en los pronombres personales.

\section{CONCLUSIONES}

Luego de analizar el corpus de este estudio, se constata que la crónica ajedrecística comparte ciertos rasgos con la crónica de otras disciplinas deportivas. Por ejemplo, la crónica ajedrecística, al igual que la crónica de otros deportes, está conformada de dos fases esenciales: descriptivo-narrativa y de comentario. Asimismo, en la fase descriptivo-narrativa, se advierte la presencia de construcciones averbales, especialmente sintagmas nominales. Igualmente, en ambas fases, el cronista ajedrecístico emplea figuras retóricas como la metonimia y la personificación. Además, se recurre a remembranzas y anécdotas que contribuyen a la amenización de la crónica.

Empero, también se constata que existen rasgos funcionales y estilísticos distintivos de la crónica ajedrecística, en especial en lo que concierne al vocabulario y las estructuras. En efecto, desde el nombre de las piezas, el sistema para describir los movimientos que se producen en el tablero, el nombre que reciben ciertas jugadas, hasta las hipótesis y suposiciones resultan rasgos distintivos de esta crónica. Consideramos que el conocimiento de estos términos y estructuras permite a los destinatarios del discurso ajedrecístico comprender la crónica de una partida sin problemas. De tal manera, concluimos que dichos términos y estructuras hacen de este discurso un sublenguaje sectorial.

Por último, la originalidad de este estudio reside en que examinamos las estructuras empleadas en un género discursivo específico, a saber: la crónica ajedrecística oral en español. Somos conscientes de que los resultados aquí presentados no pueden considerarse decisivos ni concluyentes, pues la muestra que hemos tomado en cuenta no es lo suficientemente amplia para hacerlo. De ahí que se requiera llevar a cabo más investigaciones sobre el discurso de esta disciplina deportiva, ya sea con base en crónicas orales, ya sea a partir de otros géneros discursivos como el comentario, el debate o la entrevista. 


\section{BIBLIOGRAFÍA}

Alasalmi, Teija. Problems of translating the laws of Rugby Union from English into Finnish. M. A. Thesis. Jyväskylä: University of Jyväskylä. 2014. Web. 3 may 2020.

Arias, Fidias G. Éxito deportivo de países latinoamericanos en Juegos Olímpicos y Panamericanos (1967-2008). Aproximación a las variables socioeconómicas asociadas. Tesis doctoral. Caracas: Universidad Central de Venezuela. 2012. Web. 9 may 2020.

Armañanzas, Emy y Fernando Sánchez Gómez El lenguaje de lucha en las crónicas de boxeo de Manuel Alcántara. Círculo de Lingüística Aplicada a la Comunicación, 39 (2009): 3-34. Impreso.

Augendre, Sandra, Kupšc, Anna, Boyé, Gilles y Catherine Mathon. "Live TV sports commentaries: specific syntactic structures and general constraints". In Legallois, D., Charnois, T., y Larjavaara, M. (eds.). The Grammar of Genres and Styles: From Discrete to Non-Discrete Units. Berlin/ Boston: De Gruyter Mouton. 2018: 194-218. Impreso.

Bandera Castro, Yainoris. Estudio de los componentes del proceso de entrenamiento del ajedrez categoría escolar de alto rendimiento. Arrancada, 15(27) (2015): 4958. Web. 30 jun 2020.

Blanco Hernández, Uvencio José. ¿Qué nos hubiera contestado Capablanca a la pregunta, es el ajedrez un deporte? Ciencia y Deporte, 5(1) (2020): 1-12. Web. 6 nov 2020.

Castro Odio, Lydia A. Lenguaje y béisbol en Cuba. La jiribilla, revista de cultura cubana. (2012). Web. 12 ene 2017.

Danell, Karl Johan. Chess and language. Some comparisons between two sign systems. Studia Neophilologica, 46 (1974): 3-9. Web. 7 may 2020.

Ermiş, Egemen y Necati Erilli. Analysis of Chess Grand Masters. European Journal of Physical Education and Sport Science, 3(11) (2017): 221-233. Web. 16 may 2020.

Fernández Amigo, Joaquín. Utilización de Material Didáctico con Recursos de Ajedrez para la Enseñanza de las Matemáticas. Estudio de sus efectos sobre una muestra de alumnos de $2^{\circ}$ de Primaria. Tesis Doctoral. Bellaterra: Universidad Autónoma de Barcelona. 2008. Impreso.

FIDE Handbook: http://www.fide.com/fide/handbook.html?id=171\&view=article 16 may 2020.

Gómez Torrego, Leonardo. Aspectos semánticos del lenguaje del fútbol en España. MarcoELE, 11 (2010): 150-158. Web. 15 may 2020.

Godoy, Fabián. Transmisiones deportivas. Claves prácticas para el periodista especializado. Buenos Aries: Librofutbol. 2014. Web. 7 may 2020.

Guerrero Salazar, Susana. El lenguaje deportivo, entre coloquial y literario. Isla de Arriarán, 19 (2002): 365-382. Web. 8 nov 2020.

Gutvay, Mauricio y Joaquín Fernández Amigo. Criterios de evaluación de los procesos de enseñanza y aprendizaje de un programa de ajedrez digital. Tendencias pedagógicas, 19 (2012): 131-148. Web. 28 jun 2020. 
Hernández Alonso, Néstor. El lenguaje de las crónicas deportivas. Madrid: Cátedra. 2003. Impreso.

Herrero Gutiérrez, Francisco Javier. Las retransmisiones deportivas radiofónicas en España: un análisis textual. Pangea, 3 (2012): 118-135. Web. 6 nov 2020.

Hesse, Christian. Expediciones al mundo del ajedrez. Asturias: Editorial Chessy. 2010. Impreso.

Karayev, Assylkhan Agbayevich. Specifics of chess terminology. Наука, техника и образование, 6(24) (2016): 311-314. Web. 14 jun 2020.

Kovacic, Diego María. Ajedrez en las escuelas. Una buena movida. Psiencia. Revista latinoamericana de ciencia psicológica, 4(1) (2012): 29-41. Web. 6 nov 2020.

Kowalikowa, Jadwiga. Language of sport in the context of communication and culture. Studies in Physical Culture and Tourism, 16(1) (2009): 63-67. Web. 15 may 2020.

Maksymenko, Elena Victoria. Отличительные черты англоязычного шахматного дискурса. Одеський лінгвістичний вісник, 2(5) (2015): 91-94. Web. 14 jun 2020.

Marín Montín, Joaquín. La crónica deportiva: José A. Sánchez Araujo, Ámbitos, 5(2) (2000): 241-257. Web. 7 may 2020.

Mathon, Catherine y Georges Boulakia. "Le commentaire sportif en direct : une combinatoire de différentes fonctions de la prosodie". En Yoo H.-Y. y DelaisRoussarie E. (ed.), Actes de la conférence «Interface, discours et prosodie». 2009: 287-301. Web. 5 jun 2020.

Medina Montero, José Francisco. "La metáfora en el léxico futbolístico: el caso de los participantes en español, y sus posibles equivalentes en italiano". En Luque Toro, L. (ed.). Léxico Español Actual. Venezia: Università Ca'Foscari di Venezia. 2007: 197-240. Web. 3 jun 2020

Naranjo de Arcos, Alicia. Tratamiento de la información deportiva en la prensa: la crónica como género prevalente. El caso de los encuentros de fútbol entre Real Madrid y F. C. Barcelona. Tesis doctoral. Málaga: Universidad de Málaga. 2011. Web. 18 may 2020.

Nomdedeu Rull, Antoni. La terminología del deporte en los diccionarios generales del español. Revista de Lexicografía, 9 (2003): 57-95. Web. 6 nov 2020.

Palacios Aldana, Ana Gerturdis y Inalvis Mengana Osorio. La comunicación y el lenguaje en la práctica del Ajedrez. Revista Científica de Ajedrez. "Capablanca", 1(1) (2020): 96-104. Web. 28 may 2020.

Pérez Saldanya, Manuel. El modo en las subordinadas relativas y adverbiales. En Bosque, I. y Demonte, V. (eds.). Gramática descriptiva de la lengua española. Madrid: Espasa-Calpe. 1999: 3253-3322. Impreso.

Quintero Ramírez, Sara. Rasgos sintácticos de la crónica futbolística. Revista de Investigación Lingüística, 18 (2015): 233-254. Impreso.

Quintero Ramírez, Sara. Metonimia como recurso cohesionador en el texto periodístico deportivo. ELUA, 31 (2017): 269-284. Impreso. 
Rakhimova, Dinara. Игровая терминология в художественном и профессиональном дискурсах (на материале произведений ВВ Набокова, АЕ Карпова, АБ Рошаля). Philology and Culture, 3(49) (2017). Web. 7 jun 2020.

Saiz Noeda, Belén. Notas sobre la retórica del lenguaje futbolístico. MarcoELE, 11 (2010): 196-227. Web. 15 may 2020.

Sammalvuo, Tapani. Trabajo terminológico basado en corpus: caso práctico de terminología del ajedrez. Tesis de Máster. Turku: Universidad de Turku. 2016. Web. 28 may 2020.

Santoro, Osmar. ¿Es el ajedrez un deporte?. ISDe Sports Magazine, 2(7) (2010): 1-6. Web. 30 jun 2020.

Simó, Judit. "It's Not All about the Brain: A Cross-Linguistic Exploration of Body-Part Metaphors in Chess". En 9th Conference on Conceptual Structure, Discourse, and Language (CSDL9). 2008. Web. 1 jul 2020

Vega Jiménez, Rafael Andrés. La información deportiva en Canal Sur: una aproximación a su tratamiento en la radio televisión de Andalucía. Tesis doctoral. Sevilla: Universidad de Sevilla. 2013. Web. 16 may 2020.

Veselinović, Ema. Expresión de probabilidad. Tesis doctoral. Zagreb: Universidad de Zagreb. 2019. Web. 13 may 2020. 